\title{
A Discrete-Time Chattering Free Sliding Mode Control with Multirate Sampling Method for Flight Simulator
}

\author{
Yunjie Wu, ${ }^{1}$ Youmin Liu, ${ }^{1}$ and Wulong Zhang ${ }^{2}$ \\ ${ }^{1}$ School of Automation Science and Electrical Engineering, Beihang University, Beijing 100191, China \\ ${ }^{2}$ Beijing Simulation Center National Defense Science and Technology Key Laboratory of Missile Control System Simulation, \\ Beijing 100854, China
}

Correspondence should be addressed to Youmin Liu; snakelym1985@163.com

Received 26 March 2013; Accepted 29 April 2013

Academic Editor: Yu Kang

Copyright (C) 2013 Yunjie Wu et al. This is an open access article distributed under the Creative Commons Attribution License, which permits unrestricted use, distribution, and reproduction in any medium, provided the original work is properly cited.

\begin{abstract}
In order to improve the tracking accuracy of flight simulator and expend its frequency response, a multirate-sampling-methodbased discrete-time chattering free sliding mode control is developed and imported into the systems. By constructing the multirate sampling sliding mode controller, the flight simulator can perfectly track a given reference signal with an arbitrarily small dynamic tracking error, and the problems caused by a contradiction of reference signal period and control period in traditional design method can be eliminated. It is proved by theoretical analysis that the extremely high dynamic tracking precision can be obtained. Meanwhile, the robustness is guaranteed by sliding mode control even though there are modeling mismatch, external disturbances and measure noise. The validity of the proposed method is confirmed by experiments on flight simulator.
\end{abstract}

\section{Introduction}

Flight simulator simulates the attitude of aircraft and helps the ground experiments. High precision motion control is the key of a flight simulator, which influences the accuracy of simulation experiments. Therefore, improving the tracking accuracy of flight simulator and expending its frequency response have always been a hot issue of the research in this field [1]. Traditional control methods construct the inverse model of the closed-loop system and add it into feedforward to achieve the dynamic tracking performance in a certain frequency range. However, the discrete model of a flight simulator system is often nonminimum phase, which can cause unstable pole zero cancelling, due to zero order hold. A zero phase error tracking controller (ZPETC) is proposed by Tomizuka to achieve high precision tracking by importing an approximate inverse model of the object in frequency domain [2]. The ZPETC has been widely used in servo control systems, especially in the fields of high accuracy motion control, such as machining and flight simulator $[3,4]$. The ZPETC needs the preview information of the desired output, which is not available in the flight simulator systems. Therefore, the current values of the command are used instead of preview ones. As a result, a certain amount of time delay is introduced into the system and the bandwidth of the system is limited.

To overcome the disadvantages of ZPETC, a multirate sampling method (MSM) is developed [5]. The MSM, in which a SISO object is described as a state equation of MIMO to construct the nonsingular transfer function matrixs between the state of the object and the input control value, can implement perfect tracking to discrete command points. The arithmetic has been tested in a hard disk drive system and a large-scale stage $[6,7]$. It needs to be emphasized that the perfect tracking is not available for a single sampling system theoretically because of the zero order hold. For a flight simulator system, the step that the simulation computer solves the mathematical model of the aircraft is often longer than the sampling period of the digital servo control system. Moreover, there exist a plurality of independent sampling periods in the system; that is, the system is a complex multirate sampling system. The conventional methods employ 
interpolation ways to obtain desired control command for each sample point after receiving the instruction of the simulation computer, and then the control algorithm is calculated [8]. Apparently, the interpolation solution does not use the difference between the sampling periods. On the contrary, the inconsistent sampling periods are seen as a negative factor. With the help of the MSM, the difference can be exploited sufficiently to improve the accuracy of the flight simulator in every sample point.

As a typical kind of servo motor system, the robustness against external nonlinear disturbances, time-varied characters, and modeling uncertainties is urgently required in the flight simulator system [1]. To satisfy the requirement of MSM, a robust controller is needed [5]. Sliding mode control (SMC), the popular nonlinear robust control strategy, which is theoretically invariant to model uncertainties and external disturbances under matching conditions, is very attractive for servo control systems [9-11]. A flight simulator system in a high-performance application must have fast response, preferably without overshoot, high static and dynamic accuracy, and robustness to parameter perturbations. SMC can in great deal meet those requirements. Various SMC algorithms have been devised for flight simulator control such as a terminal sliding mode method [12], an adaptive sliding mode method [13], and a fuzzy sliding mode method [14].

Unfortunately, the SMC also causes chattering phenomenon while inhibiting disturbance by switching control value. Chattering is a serious impediment for SMC application. The MSM helps to improve the dynamic performance of the sliding mode controller; on the other hand, it makes the system more sensitive for chattering. Therefore, a chattering free sliding mode controller is needed to combine with the MSM. The SMC is designed using the algorithm in [15]. The control law obtained from the reaching law has two modes: a nonlinear and a linear mode. The nonlinear mode steers the system to a vicinity of the sliding manifold, and the linear mode ensures the sliding manifold is reached in one step and maintains the motion on it after that. The algorithm has been used in induction motor systems [16, 17]. However, the unsatisfactory tracking accuracy limits the application of the theory.

In this paper, a discrete-time chattering free sliding mode control (DSMC) with MSM is proposed. The multirate sampling part helps to improve the dynamic tracking accuracy and expend the frequency response, while the sliding mode part helps to enhance the robustness when there exists large nonlinear factors and modeling mismatch. Moreover, the resonance caused by sensitive MSM in controlling systems with chattering, which is restrained by algorithm, can be inhibited. The proposed method comprehended the advantages of both MSM and DSMC.

The brief outline of the paper is as follows. In Section 2, the multirate sampling method is introduced. In Section 3, the discrete-time chattering free sliding mode control method is proposed. In Section 4, experiments results are included to support the theoretical work. Finally, the paper is concluded in Section 5.

\section{Multirate Sampling Method}

For a flight simulator system, the command transmission period of the simulation computer $T_{r}$ is ordinarily longer than the sampling period $T_{s}$ of the control system. The interpolation algorithm calculates the desired control command value at every point between $i T_{r}$ and $(i+1) T_{r}$. In the analysis of MSM, a single sampling SISO system is described as an MIMO system. Therefore, the interpolation is not required to calculate the commands. Figure 1 shows the structure of a multirate sampling control system.

In the structure, $C_{M}(z)$ guarantees the tracking performance and $C_{R}\left(z_{s}\right)$ improves the robustness. $C_{M}(z)$ is a feedforward MIMO controller. As is shown in (1), $L\left(T_{s}\right)$, an MISO component, outputs each element $u_{k}[i]$ of the input vector $\overrightarrow{\mathbf{u}}[i]$ in accordance with the sampling period $T_{s} . C_{R}\left(z_{s}\right)$ is a robust controller, which is used to restrain external nonlinear disturbances, time-varied characters, and modeling uncertainties. $P_{c}(s)$ is the continuous-time object. $S_{M}$ denotes sampling. $H_{M}$ denotes zero order hold. For a general multirate sampling system, there exist three periods: the reference input period $T_{r}$, the control value input period $T_{u}$, and the feedback sampling period $T_{y}$. In flight simulator systems, the previous periods satisfy (2). Consequently, the system can be divided into two parts: the shorter period part with $T_{s}$ and the longer period part with $T_{r}$.

Suppose that the state space model with controllable standard of the flight simulator system in work frequency band is shown as (3). Then the discrete-time plant discretized by sampling period $T_{s}$ can be gotten as $(4)(\bullet(k)$ stands for $\bullet(k T))$

$$
\begin{aligned}
& \overrightarrow{\mathbf{u}}[i]= {\left[u_{1}[i], u_{2}[i], \ldots, u_{k}[i], \ldots, u_{n}[i]\right]^{T}, } \\
& L\left(T_{s}\right) \overrightarrow{\mathbf{u}}[i]= \begin{cases}u_{1}[i], & t=T_{s}, \\
u_{2}[i], & t=2 T_{s}, \\
\vdots & \\
u_{k}[i], & t=k T_{s}, \\
\vdots & \\
u_{n}[i], & t=n T_{s},\end{cases} \\
& T_{r}>T_{u}=T_{y}=T_{s}, \\
& \dot{\mathbf{x}}(t)=A_{c} \mathbf{x}(t)+b_{c} u(t), \\
& y(t)=c_{c} \mathbf{x}(t), \\
& \mathbf{x}[k+1]=A_{s} \mathbf{x}[k]+b_{s} u[k], \\
& y[k]=c_{s} \mathbf{x}[k] .
\end{aligned}
$$

In the following discussions, $T_{r}=n T_{s}$ is regarded as the condition, which is very common in the flight simulator systems. In this equation, $n$ is the quantity of state variables of the plant, that is, the plant order. Therefore, the state equation of the system (5) and (6), discretized by sampling period $T_{s}$, can be described as (7) according to $T_{r}$. It should be 


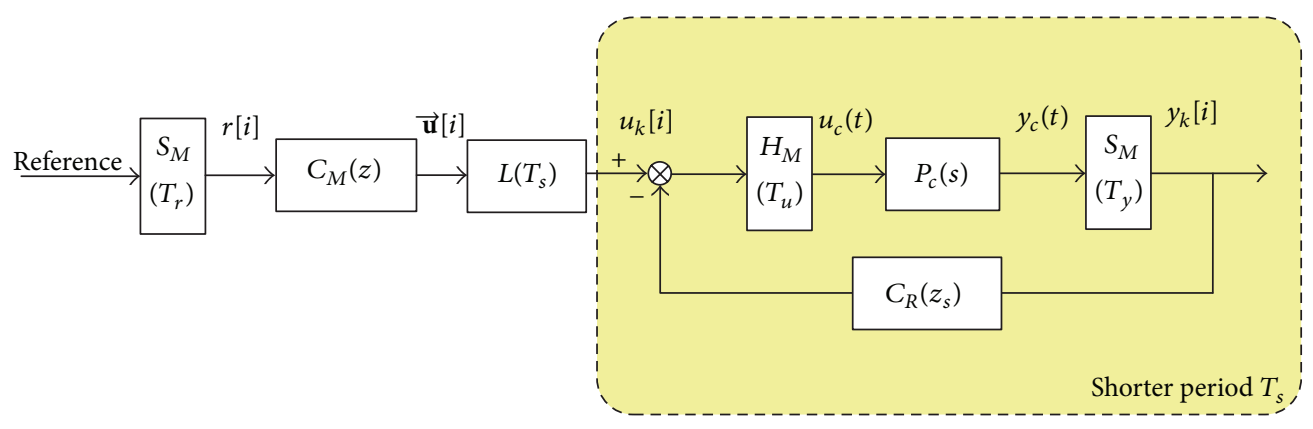

FIGURE 1: Structure of a multirate sampling control system.

emphasized that the system is described, not discretized by $T_{r}$, as

$$
\begin{gathered}
\mathbf{x}_{2}[i]=A_{s} \mathbf{x}_{1}[i]+b_{s} u_{1}[i], \\
\mathbf{x}_{3}[i]=A_{s} \mathbf{x}_{2}[i]+b_{s} u_{2}[i] \\
=A_{s}^{2} \mathbf{x}_{1}[i]+A_{s} b_{s} u_{1}[i]+b_{s} u_{2}[i], \\
\vdots \\
\mathbf{x}_{n}=[i] A_{s}^{n-1} \mathbf{x}_{1}[i]+A_{s}^{n-2} b_{s} u_{1}[i] \\
+\cdots+b_{s} u_{n-1}[i] \\
y_{1}[i]=c_{s} \mathbf{x}_{1}[i] \\
y_{2}[i]=c_{s} \mathbf{x}_{2}[i]=c_{s} A_{s} \mathbf{x}_{1}[i]+c_{s} b_{s} u_{1}[i] \\
\vdots \\
y_{n}[i]=c_{s} A_{s}^{n-1} \mathbf{x}_{1}[i]+c_{s} A_{s}^{n-2} b_{s} u_{1}[i] \\
+\cdots+c_{s} b_{s} u_{n-1}[i] \\
\mathbf{x}[i+1]=A \mathbf{x}[i]+B \overrightarrow{\mathbf{u}}[i] \\
\overrightarrow{\mathbf{y}}[i]=C \mathbf{x}[i]+D \overrightarrow{\mathbf{u}}[i],
\end{gathered}
$$

where $\overrightarrow{\mathbf{u}}[i]$ is as shown in (1), $\overrightarrow{\mathbf{y}}[i]$ is shown as (8), and $A, B, C$, $D$ are shown as (9), where

$$
\begin{aligned}
& \overrightarrow{\mathbf{y}}[i]=\left[y_{1}[i], y_{2}[i], \ldots, y_{k}[i], \ldots, y_{n}[i]\right]^{T}, \\
& {\left[\begin{array}{c|c}
A & B \\
\hline C & D
\end{array}\right]=\left[\begin{array}{c|ccccc}
A_{s}^{n} & A_{s}^{n-1} b_{s} & A_{s}^{n-2} b_{s} & \cdots & A_{s} b_{s} & b_{s} \\
\hline c_{s} & 0 & 0 & \cdots & 0 & 0 \\
c_{s} A_{s} & c_{s} b_{s} & 0 & \cdots & 0 & 0 \\
\vdots & \vdots & \vdots & & \vdots & \vdots \\
c_{s} A_{s}^{n-1} & c_{s} A_{s}^{n-2} b_{s} & c_{s} A_{s}^{n-3} b_{s} & \cdots & c_{s} b_{s} & 0
\end{array}\right] . }
\end{aligned}
$$

If the external nonlinear disturbances, time-varied characters, and modeling uncertainties are ignored, (10) and (11) can be gotten from (7). $\mathbf{x}_{d}[i+1]$ in (11) is the desired state of the system at the next time point. Consequently, with the control value (11), the system can achieve perfect tracking to the reference as is shown in (12), where

$$
\begin{gathered}
\left(I-z^{-1} A\right) \mathbf{x}[i+1]=B \overrightarrow{\mathbf{u}}[i], \\
\overrightarrow{\mathbf{u}}[i]=B^{-1}\left(I-z^{-1} A\right) \mathbf{x}_{d}[i+1], \\
\mathbf{x}[i]=\mathbf{x}_{d}[i] .
\end{gathered}
$$

However, there exist disturbance factors in real systems, which influence the control effect. Therefore, a robust controller $C_{R}\left(z_{s}\right)$ is necessary in practical application to guarantee that the sensitivity of the system to the disturbance factors is sufficiently small. Considering the robust controller, the feed forward in MSM can be described as (13). $C_{M}(z)$ is a pulse transfer function matrix with $n$-input and $n$-output. In this paper, a discrete-time chattering free sliding mode controller is employed as the robust controller as

$$
\begin{aligned}
C_{M}(z)= & B^{-1}\left(I-z^{-1} A\right)+C_{R}\left(z_{s}\right) \\
& \times\left(z^{-1} C+D B^{-1}\left(I-z^{-1} A\right)\right) .
\end{aligned}
$$

Considering the previous disturbance factors, Figure 1 can be transformed to Figure 2 from (13). In Figure 2, $C_{M 0}(z)=$ $B^{-1}\left(I-z^{-1} A\right), P\left(z_{s}\right)=P_{n}\left(z_{s}\right)\left[1+\Delta\left(z_{s}\right)\right]$ is the nominal model considering multiplicative perturbation, $d_{\mathrm{ex}}$ is the external disturbance torque, and $d$ is the equivalent disturbance, which is treated by the DSMC in this paper.

\section{Discrete-Time Sliding Mode Control Design}

Consider the continuous-time equation described by (3). The flight simulator system is a two-order servo motor control system, and the state parameters are usually defined as $x_{1}=$ $\theta, x_{2}=\dot{\theta}=\omega$ (angular position and angular velocity). Therefore, (14) can be gotten, where $J$ is the equivalent inertia, and $B$ is the equivalent damping. It is convenient and intuitionistic to transform the system model into canonical tracking error space as the control objective is to make the response track the reference. Equation (15) is gotten with this 
thinking, where $e_{1}=r-\theta=r-x_{1}, e_{2}=\dot{e}_{1}=\dot{r}-\dot{\theta}=\dot{r}-x_{2}$ and $\xi_{r}=-\ddot{r}+a \dot{r}$; as

$$
\begin{gathered}
\dot{\mathbf{x}}(t)=A_{c} \mathbf{x}(t)+b_{c} u(t), \\
y(t)=x_{1}, \\
A_{c}=\left[\begin{array}{ll}
0 & 1 \\
0 & a
\end{array}\right], \quad b_{c}=\left[\begin{array}{l}
0 \\
b
\end{array}\right], \quad a=-\frac{B}{J}, \quad b=\frac{1}{J}, \\
\dot{e}=A_{c} e-b_{c}\left(u+b^{-1} \xi_{r}\right) .
\end{gathered}
$$

The additional disturbance $b_{c} b^{-1} \xi_{r}$ appears due to the transformation, while the reference signal varies in time. In the flight simulator system, $\xi_{r}$ can be ignored in the static condition as the reference almost has no change. Meanwhile, $\xi_{r}$ can also be compensated with the help of the MSM in the dynamic condition. Consequently, the influence of $\xi_{r}$ can be ignored in the proposed method. Equation (15) can be transferred to (16). The equivalent discrete-time representation of (15) is described by (17), and the state matrices of the system have a relationship as shown in (18), where

$$
\begin{aligned}
\dot{e} & =A_{c} e-b_{c} u, \\
e[k+1] & =A_{s} e[k]-b_{s} u[k] \\
A_{s} & =e^{A_{c} T_{s}}, \quad b_{s}=\int_{0}^{T_{s}} e^{A_{c} \tau} b_{c} d \tau .
\end{aligned}
$$

It is necessary to establish a discrete-time sliding mode along the sliding surface defined by (19), where $c \in \mathfrak{R}^{1 \times 2}$. With the appropriate selection of the vector $c$, the sliding dynamics can be stable and the ideal tracking can be achieved. Consider

$$
s[k]=c \mathbf{e}[k] .
$$

The chattering free sliding mode control algorithm combines two SMC principles: the reaching law and the boundary layer. The control law has two modes: a nonlinear and a linear mode. The nonlinear mode steers the system to a boundary layer of the sliding surface, and the linear mode ensures the sliding surface is reached in one step and maintains the motion on it after that.

The reaching law is designed by (20) and (21) as follows:

$$
\begin{gathered}
s[k+1]=s[k]-\phi(s[k]), \\
\phi(s[k])=\min \left(|s[k]|, \sigma T_{s}\right) \operatorname{sgn}(s[k]) .
\end{gathered}
$$

If $|s[k]| \geq \sigma T_{s}$ (outside the boundary layer), (20) equals to (22). Finite-time convergence to the boundary layer is guaranteed in the case of $\sigma>0$ as

$$
s[k+1]=s[k]-\sigma T_{s} \operatorname{sgn}(s[k]) .
$$

If $|s[k]|<\sigma T_{s}$ (inside the boundary layer), (20) equals to $s[k+$ $1]=0$, indicating that an ideal DSM is achieved in one step.

Assume that $c b_{s}=-T_{s}$; from (17)-(21), the control law is determined as (23). The assumption ensures that the degree of variable $s$, with respect to the control signal $u$, is one, as the usual practical condition

$$
\begin{aligned}
u_{s}[k] & =\left(c b_{s}\right)^{-1} c\left(A_{s}-I\right) e[k]+\left(c b_{s}\right)^{-1} \phi(s[k]) \\
& =-\frac{c\left(A_{s}-I\right) e[k]}{T_{s}}-\frac{\phi(s[k])}{T_{s}} .
\end{aligned}
$$

Vector $c$ in (19) should be designed to ensure the exponential convergence of the DSMC, with a desired rate $\delta_{1}=e^{-\alpha T_{s}}(\alpha>$ $0)$. The system (16) with control (23) is transformed into a regular form by the coordinate transformation $e[k]=P_{1} \widetilde{e}[k]$, where

$$
\begin{gathered}
P_{1}=\left[\begin{array}{ll}
b_{s} & A_{s} b_{s}
\end{array}\right]\left[\begin{array}{cc}
a_{1} & 1 \\
1 & 0
\end{array}\right], \\
\operatorname{det}\left(z I-A_{s}\right)=z^{2}+a_{1} z+a_{0} .
\end{gathered}
$$

Since the pair $\left(A_{c}, b_{c}\right)$ is controllable and $\left(A_{s}, b_{s}\right)$ is the analytic functions of $T_{s}$, the pair $\left(A_{s}, b_{s}\right)$ is controllable for almost all choices of $T_{s}$. Therefore, the matrix $P_{1}$ is regular. Under the assumption that $c b_{s}=-T_{s}$, the vector $c$, providing the desired convergence dynamics, can be obtained as

$$
c=T_{s}\left[\delta_{1}-1\right] P_{1}^{-1} .
$$

To improve the static accuracy, a specific integral action is introduced, which is described in [18]. The integral action only effects inside the boundary layer during the linear control mode, without any degradation of the system dynamics. The control law is enhanced as (26), where the integral action is given by (27), where

$$
\begin{gathered}
u[k]=u_{s}[k]-u_{I}[k] \\
u_{I}[k]=\left\{\begin{array}{ll}
0, & |s[k]| \geq \sigma T_{s}, \\
h s[k]+u_{I}[k-1], & |s[k]|<\sigma T_{s},
\end{array} \quad 0<h<\frac{1}{T_{s}} .\right.
\end{gathered}
$$

In the proposed method, the DSMC is employed as a robust controller, which guarantees the capability of robustness [15]. The tracking performance is guaranteed by the MSM. Therefore, the static characters are considered primarily. The DSMC is designed in the static condition and $\xi_{r}$ in (15) can be ignored. In Figure 2, the DSMC works as the robust controller $C_{R}\left(z_{s}\right) \cdot C_{R}\left(z_{s}\right)$ can be expressed as $C_{R}(z)$ by $T_{r}$, using a similar way as shown in (5) and (6).

\section{Experiment Results}

In order to test the effect of the proposed method, an experiment is implemented by using a three-axis flight simulator shown in Figure 3. The optical-electrical encoder with resolution of 0.0007 degrees is employed as the position sensor. The program of control algorithm is written with $\mathrm{C}$ language based on Windows-RTX real-time system in an industrial computer (Advantech IPC 610), which connects with the servo drivers by a 16-bit D/A convertor of PCI bus. 


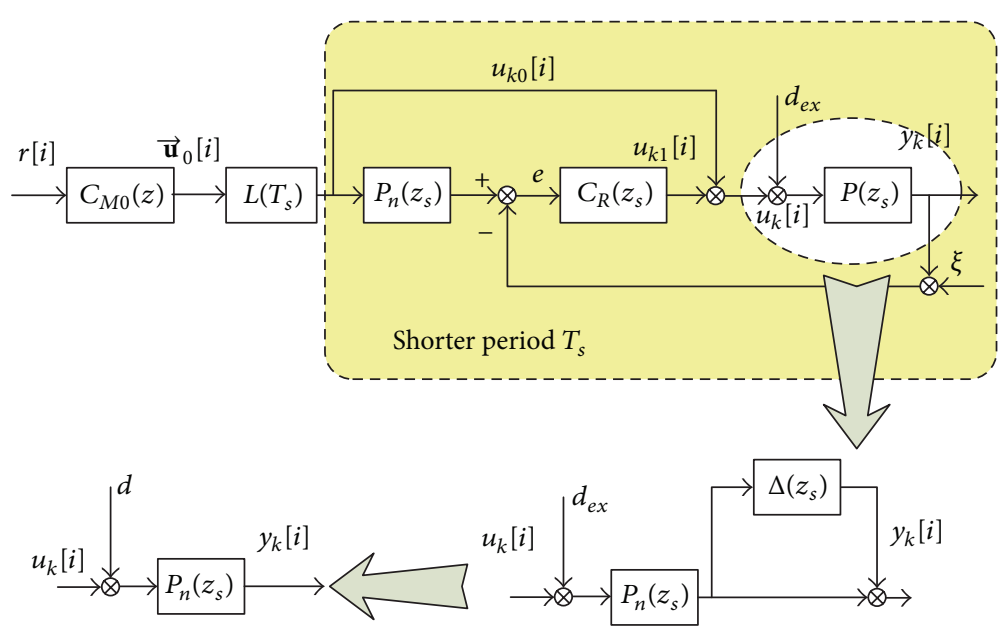

FIGURE 2: Transformed structure of the multirate sampling control system.

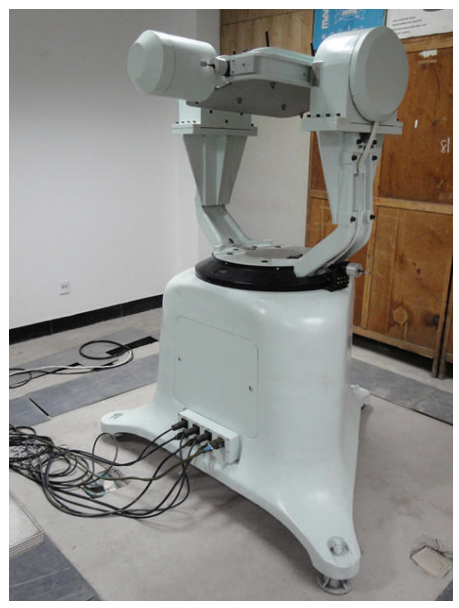

FIgURE 3: The three-axis flight simulator.

The pitch axis is chosen herein to verify the method as each axis of the flight simulator can be designed independently. The controller design is based on the parameters which are acquired by identifying the flight simulator. The parameters of the nominal model are identified as $J_{n}=$ $0.0053, B_{n}=0.0638$. The fitting curves for frequency characteristics of actual plant and nominal model shown in Figure 4. Suppose that the instruction transmission period (longer period) of the simulation computer is $T_{r}=0.002 \mathrm{~s}$. Considering $T_{r}=n T_{s}$, the control period (shorter period) is $T_{s}=0.001 \mathrm{~s}$ as the nominal model is a two-order system. Noting that the perfect tracking can be achieved no matter how to select state variables in theory, however, there exists volatility in the controller output if improper state variables are chosen in application. State variables with explicit physical significance are appropriate in general.

From (9) and (18), the multirate sampling controller in Figure 2 can be designed as

$$
C_{M 0}(z)=B^{-1}\left(I-z^{-1} A\right),
$$

where

$$
A=\left[\begin{array}{ll}
1 & 0.0020 \\
0 & 0.9763
\end{array}\right], \quad B^{-1}=\left[\begin{array}{cc}
5351.1 & -2.7 \\
-5287.3 & 8
\end{array}\right] .
$$

The DSMC is designed as (20), (21), (23), (26), and (27). The parameters of the DSMC controller are given as follows: the switching gain $\sigma=35$, and the integral gain $h=10$, the dynamics eigenvalue of sliding mode $\delta_{1}=e^{-0.02}(\alpha=20)$, and the switching function vector $c=\left[\begin{array}{lll}-0.1061 & -0.0053\end{array}\right]$. A nonlinear tracking differential estimator is used to get differential value [19], and the nonlinear tracking differential gain $g_{v}=350$. In the experiment, three sinusoidal signals with the same amplitude $0.5^{\circ}$, which is provided by the flight simulator testing standard, are chosen as the reference. The frequency of the signals are $1 \mathrm{HZ}, 2 \mathrm{HZ}$, and $4 \mathrm{HZ}$, respectively. An independent DSMC, with the control cycle $T_{s}=0.001 \mathrm{~s}$, is designed aiming at the same object for comparison. For the proposed MSM, the references are as (30)-(32), respectively, where

$$
\begin{aligned}
& r[i]=\mathbf{x}_{d}[i+1]=\left[\begin{array}{c}
0.5 \sin (2 \pi i) \\
\pi \cos (2 \pi i)
\end{array}\right], \\
& r[i]=\mathbf{x}_{d}[i+1]=\left[\begin{array}{c}
0.5 \sin (4 \pi i) \\
2 \pi \cos (4 \pi i)
\end{array}\right], \\
& r[i]=\mathbf{x}_{d}[i+1]=\left[\begin{array}{l}
0.5 \sin (8 \pi i) \\
4 \pi \cos (8 \pi i)
\end{array}\right] .
\end{aligned}
$$

Figure 5 compares the tracking error of the DSMC and DSMC+MSM schemes when the reference signal is described as $0.5 \sin (2 \pi t)$. The curves of the tracking error show that the maximum tracking error under the DSMC scheme approximately equals to $0.018 \mathrm{deg}$ while that decreases to $0.005 \mathrm{deg}$ using the proposed method. Figure 6 compares the tracking error of the two schemes when the reference signal is described as $0.5 \sin (4 \pi t)$. The maximum tracking error decreases from $0.022 \mathrm{deg}$ to $0.012 \mathrm{deg}$ with the help of MSM. Figure 7 compares the tracking error of the two schemes when the reference signal is described as 


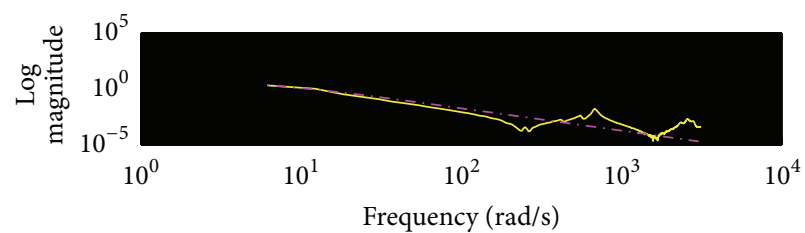

(a)

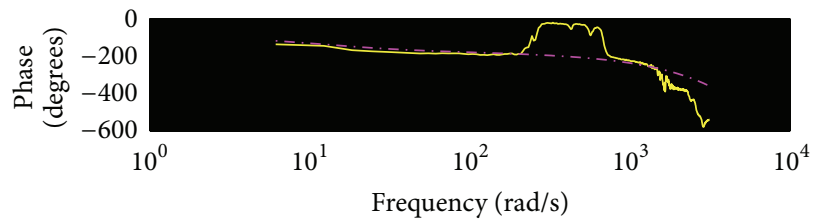

(b)

FIGURE 4: The fitting curves for frequency characteristics of actual plant and nominal model.

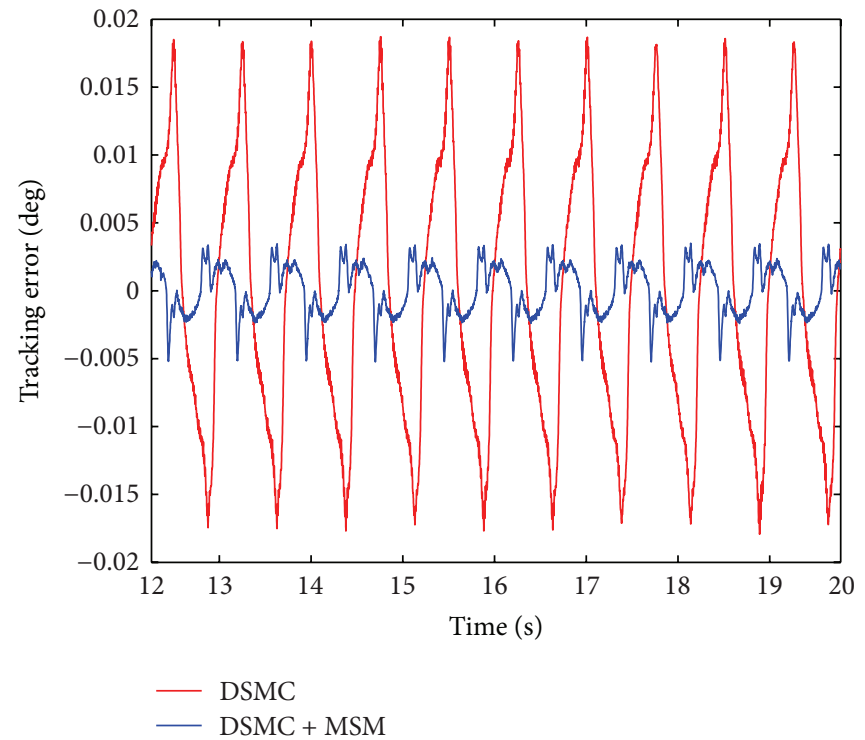

(a)

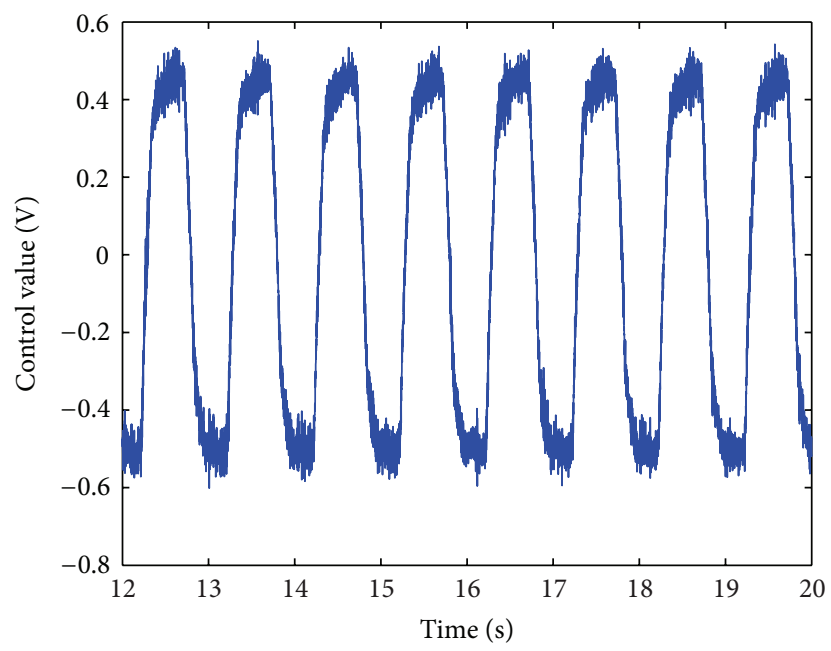

(b)

FIGURE 5: The comparison of error curves with the sinusoidal input $(A=0.5, f=1)$ and the control value of the proposed method.

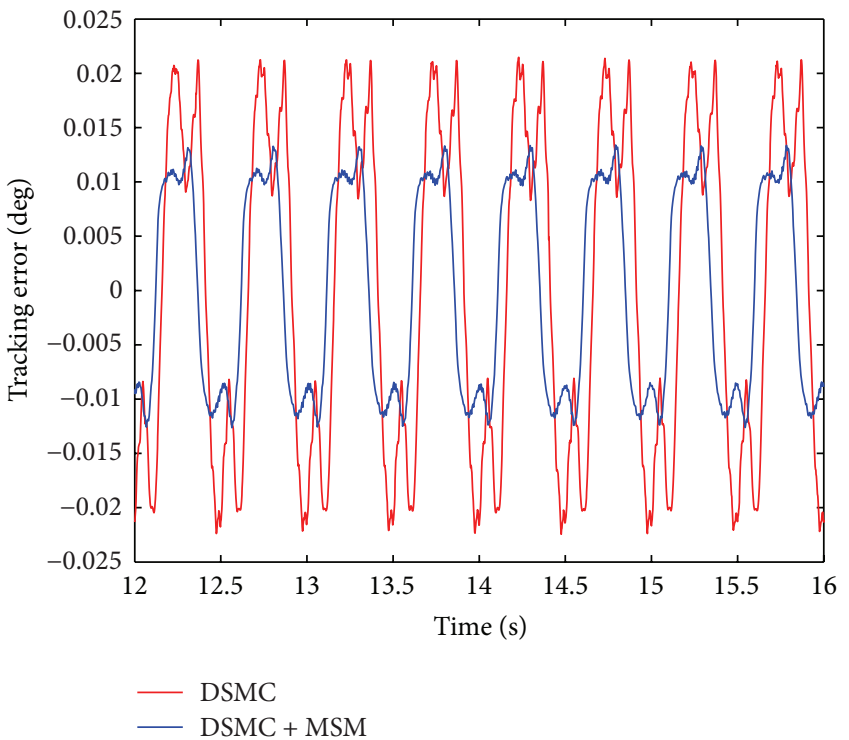

(a)

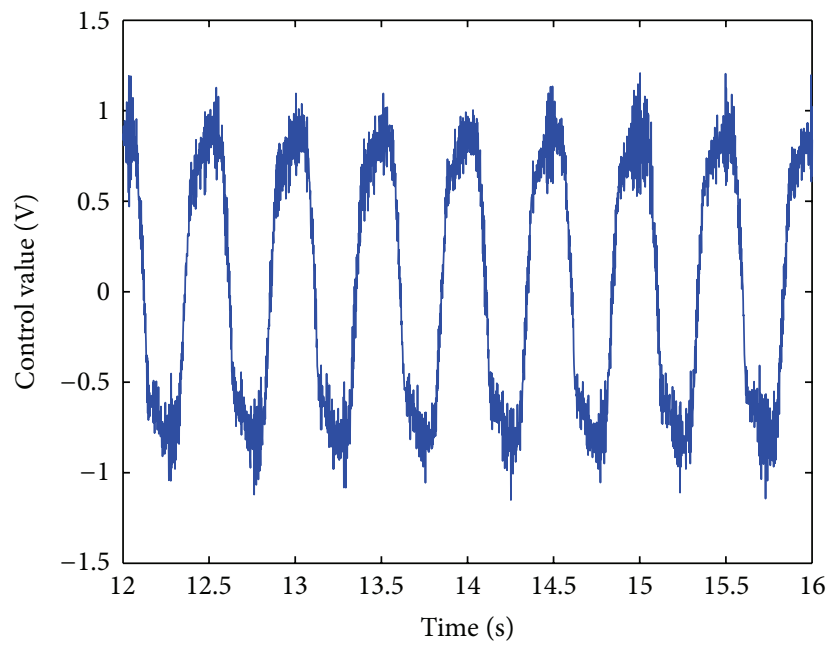

(b)

Figure 6: The comparison of error curves with the sinusoidal input $(A=0.5, f=2)$ and the control value of the proposed method. 


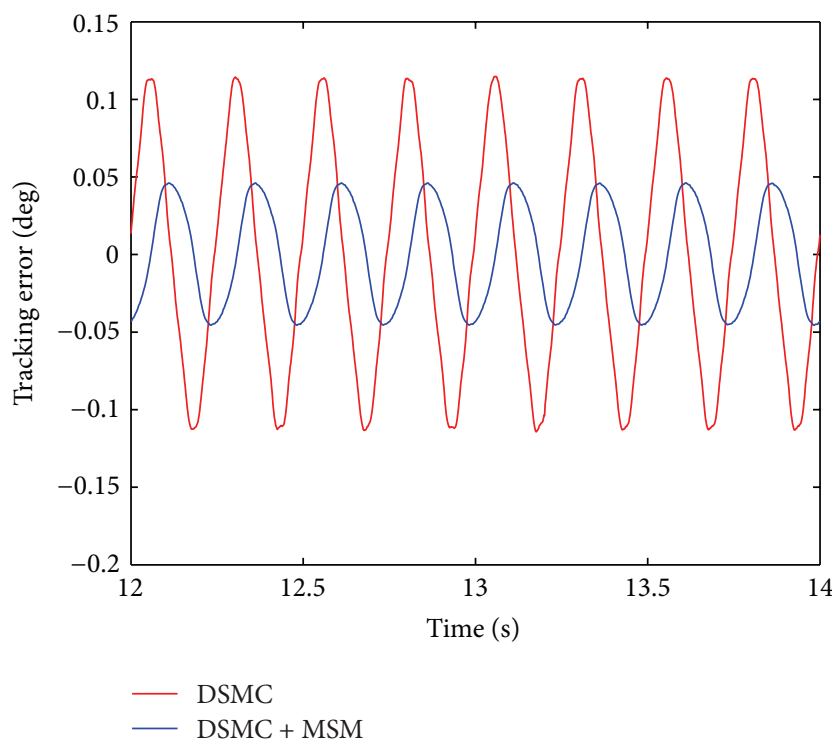

(a)

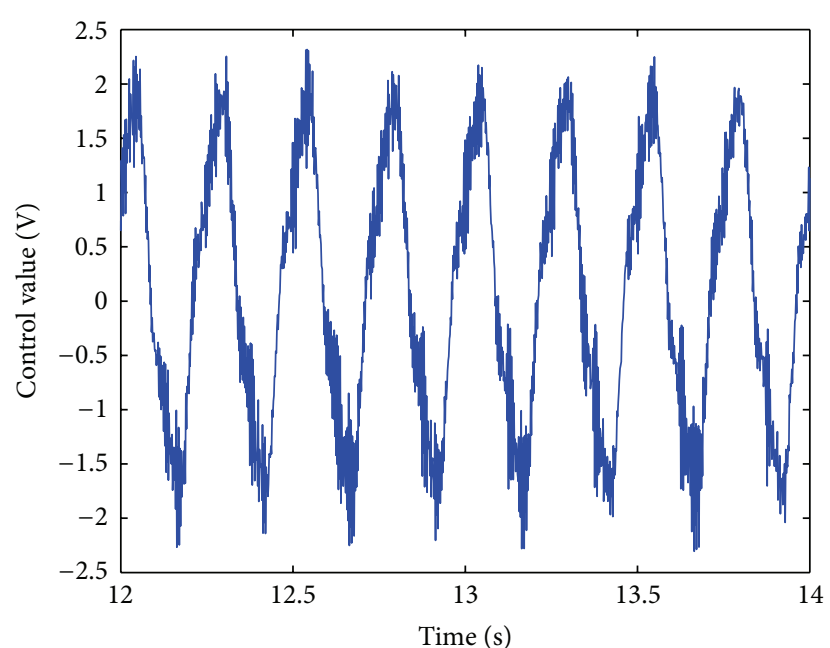

(b)

FIGURE 7: The comparison of error curves with the sinusoidal input $(A=0.5, f=4)$ and the control value of the proposed method.

$0.5 \sin (8 \pi t)$. The maximum tracking error decreases from 0.114 deg to $0.046 \mathrm{deg}$.

The tracking error indicates that the system with DSMC+ MSM has better dynamic performance than the system with independent DSMC. The flight simulator testing standard stipulates that the maximum tracking error cannot exceed $10 \%$ of the amplitude of the test reference in working band. Consequently, the maximum dynamic tracking error should be less than $0.05 \mathrm{deg}$ under the condition of the standard testing reference shown in (30)-(32). From Figure 7, the system using the proposed method has a bandwidth which is more than $4 \mathrm{HZ}$. Meanwhile, the bandwidth of the same system using the independent DSMC is less than $4 \mathrm{HZ}$. The frequency response of the flight simulator is expended. Meanwhile, the controller is designed in accordance with the nominal model, without considering external disturbance, modeling mismatching, and measure noise. The experiment results got by a real system with previous disturbances are similar to the theoretical ones, which intimates the robustness of the method.

Furthermore, the control value of the proposed method does not exhibit obvious chattering phenomenon from the control value curves in Figures 5-7. The control value does not exceed the limit of the D/A converter either. External disturbance, modeling mismatch, measure noise, and other factors are reflected in control value due to the effect of robust controller, which is a DSMC in this paper. The results indicate that the introduced controller can be reliably performed in practical application.

\section{Conclusion}

This paper proposes a discrete-time chattering free sliding mode control with multirate sampling method. The DSMC is employed as a robust controller in the MSM structure. The
DSMC compensates the nonlinear disturbance and ensures the robustness of the system. The chattering is eliminated by algorithm. Meanwhile, the MSM helps to improve the dynamic performance and expend the frequency response of the system. In consequence, they help each other to enhance the performance of the servo control system.

The method has been validated by experiments. By using the proposed method, the maximum tracking error decreases from $0.018 \mathrm{deg}$ to $0.005 \mathrm{deg}$ with the reference of $0.5 \sin (2 \pi t)$, from $0.022 \mathrm{deg}$ to $0.012 \mathrm{deg}$ with the reference of $0.5 \sin (4 \pi t)$, and from $0.114 \mathrm{deg}$ to $0.046 \mathrm{deg}$ with the reference of $0.5 \sin (8 \pi t)$. Most servo motion control systems have similar characteristics to flight simulator therefore, the theoretic results are able to be extended to other relational fields such as mechanical arm systems, camera tracking systems, and other servo motion control systems, especially those with high precision and frequency response requirement.

However, the restriction that $T_{r}=n T_{s}$ must be satisfied, where $n$ is the order of the plant. In practical application, the change of the sampling period means additional debugging workload frequently. In future work, better ways to achieve multirate sampling will be studied.

\section{Acknowledgment}

This work was supported by the National Natural Science Foundation of China (Grant no. 91216304).

\section{References}

[1] Y. Liu, Y. Deng, and D. Tian, "Compound disturbance observer for flight simulator," Communications in Computer and Information Science, vol. 325, pp. 197-205, 2012. 
[2] M. Tomizuka, "Zero phase error tracking algorithm for digital control," ASME Journal of Dynamic Systems, Measurement and Control, vol. 190, no. 1, pp. 65-68, 1987.

[3] J. Liu, P. He, and L. Er, "Zero phase error control based on neural compensation for flight simulator servo system," Journal of Systems Engineering and Electronics, vol. 17, no. 4, pp. 793-797, 2006.

[4] A. Ramli, I. Hashimah, I. Norlela, T. Mazidah, and R. M. H. Fazalul, "Real-time adaptive feedforward zero phase error tracking control for X-Y motion," in Proceedings of the International Conference on System Engineering and Technology (ICSET '12), September 2012.

[5] H. Fujimoto, Y. Hori, and A. Kawamura, "Perfect tracking control based on multirate feedforward control with generalized sampling periods," IEEE Transactions on Industrial Electronics, vol. 48, no. 3, pp. 636-644, 2001.

[6] H. Fujimoto, K. Fukushima, and S. Nakagawa, "Vibration suppression short-span seeking of HDD with multirate feedforward control," in Proceedings of the American Control Conference, pp. 582-587, Minneapolis, Minn, USA, June 2006.

[7] K. Saiki, A. Hara, K. Sakata, and H. Fujimoto, "A study on high-speed and high-precision tracking control of largescale stage using perfect tracking control method based on multirate feedforward control," IEEE Transactions on Industrial Electronics, vol. 57, no. 4, pp. 1393-1400, 2010.

[8] Z. Yao, W. Wang, and L. Zheng, "A hybrid adaptive control for flight simulator turn table," in Proceedings of the IEEE International Conference on Mechatronics and Automation (ICMA '10), pp. 773-777, August 2010.

[9] Q. Khan, A. I. Bhatti, M. Iqbal, and Q. Ahmed, "Dynamic integral sliding mode control for SISO uncertain nonlinear systems," International Journal of Innovative Computing, Information and Control A, vol. 8, no. 7, pp. 4621-4633, 2012.

[10] J. Yang, S. Li, and X. Yu, "Sliding-mode control for systems with mismatched uncertainties via a disturbance observer," IEEE Transactions on Industrial Electronics, vol. 60, no. 1, pp. 160-169, 2013.

[11] Z. Qiao, T. Shi, Y. Wang, Y. Yan, C. Xia, and X. He, "New slidingmode observer for position sensorless control of permanentmagnet synchronous motor," IEEE Transactions on Industrial Electronics, vol. 60, no. 2, pp. 710-719, 2013.

[12] T. Karbasi, A. Ghahramani, A. Kashaninia, and A. Vali, "Sliding and terminal sliding mode control of a 3 -axis flight motion simulator," in Proceedings of the 20th Iranian Conference on Electrical Engineering (ICEE '12), pp. 934-939, May 2012.

[13] L. Hui-Bo and Z. Hai-Jing, "The backstepping adaptive sliding mode control for the flight simulator servo system," in Proceedings of the 30th Chinese Control Conference (CCC '11), pp. 25332538, July 2011.

[14] W. Yunjie, L. Weiting, and L. Youmin, "Fuzzy sliding mode control based on optimal fuzzy reasoning and its application on simulator turntable," in Proceedings of the Asia Simulation Conference-7th International Conference on System Simulation and Scientific Computing (ICSC '08), pp. 765-769, October 2008.

[15] G. Golo and C. Milosavljević, "Robust discrete-time chattering free sliding mode control," Systems and Control Letters, vol. 41, no. 1, pp. 19-28, 2000.

[16] B. Veselić, B. Peruničić-Draženović, and C. S. Milosavljević, "High-performance position control of induction motor using discrete-time sliding-mode control," IEEE Transactions on Industrial Electronics, vol. 55, no. 11, pp. 3809-3817, 2008.
[17] B. Veselić, B. Peruničić-Draženović, and C. S. Milosavljević, "Improved discrete-time sliding-mode position control using Euler velocity estimation," IEEE Transactions on Industrial Electronics, vol. 57, no. 11, pp. 3840-3847, 2010.

[18] C. Milosavljević, B. Perunicic-Drazenovic, B. Veselic, and D. Mitic, "A new design of servomechanisms with digital sliding mode," Electrical Engineering, vol. 89, no. 3, pp. 233-244, 2007.

[19] D. Tian, D. Yashiro, and K. Ohnishi, "New nonlinear tracking differential-estimators: theory and practice," International Journal of Innovative Computing, Information and Control, vol. 8, no. 12, pp. 8257-8271, 2012. 


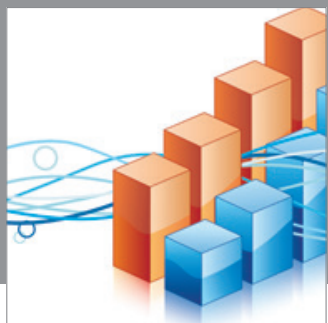

Advances in

Operations Research

mansans

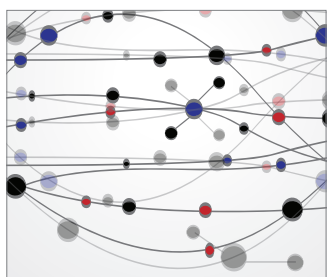

The Scientific World Journal
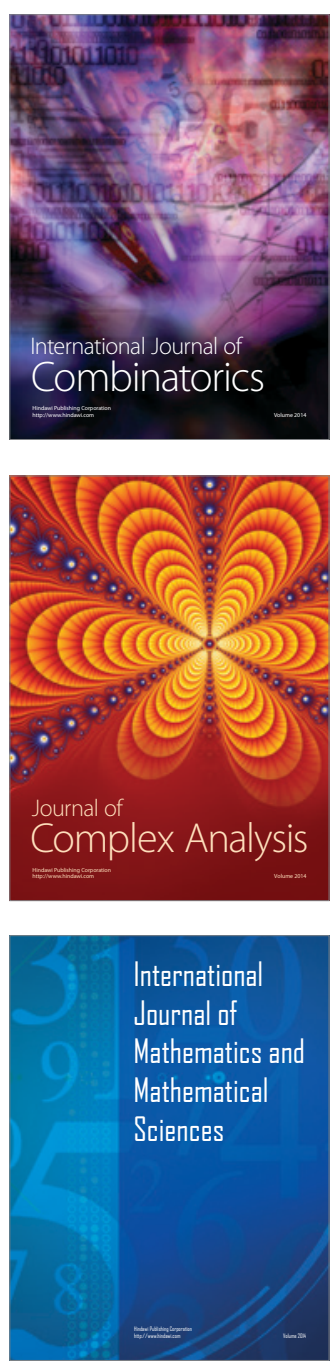
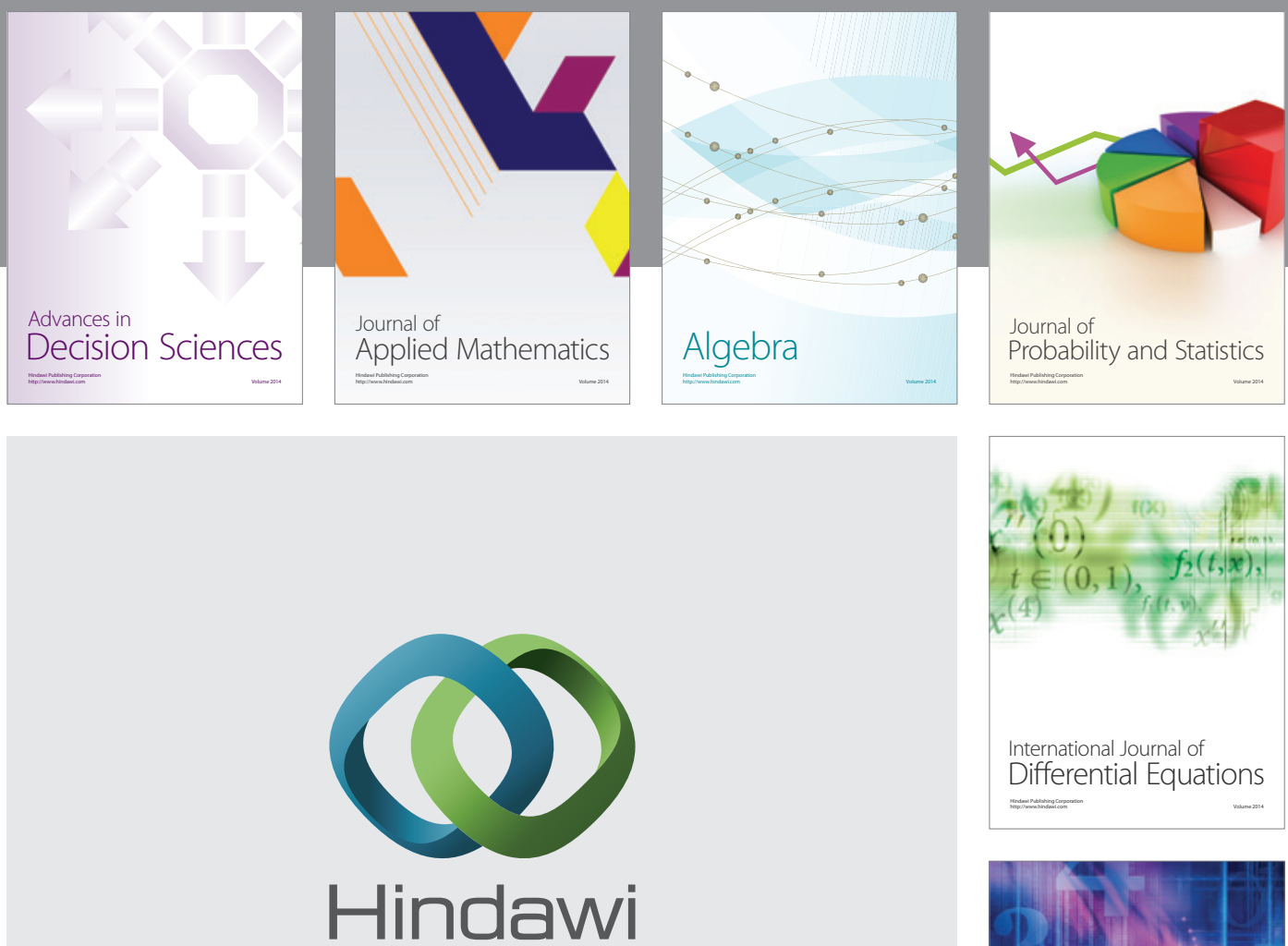

Submit your manuscripts at http://www.hindawi.com
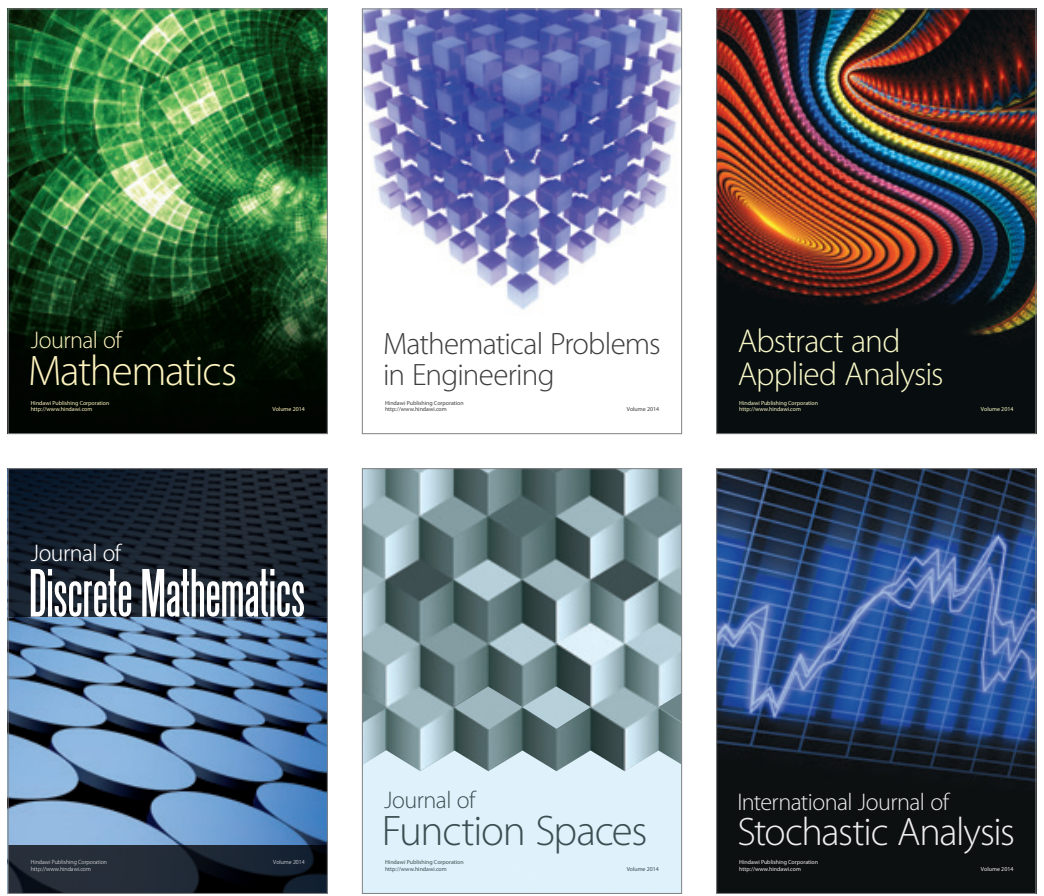

Journal of

Function Spaces

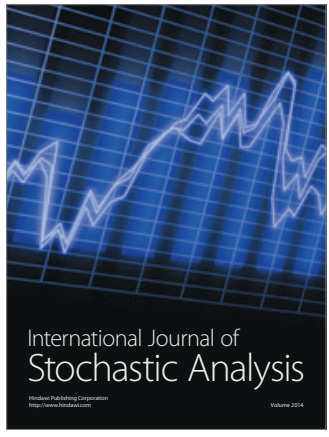

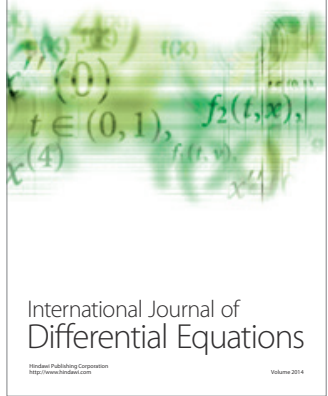
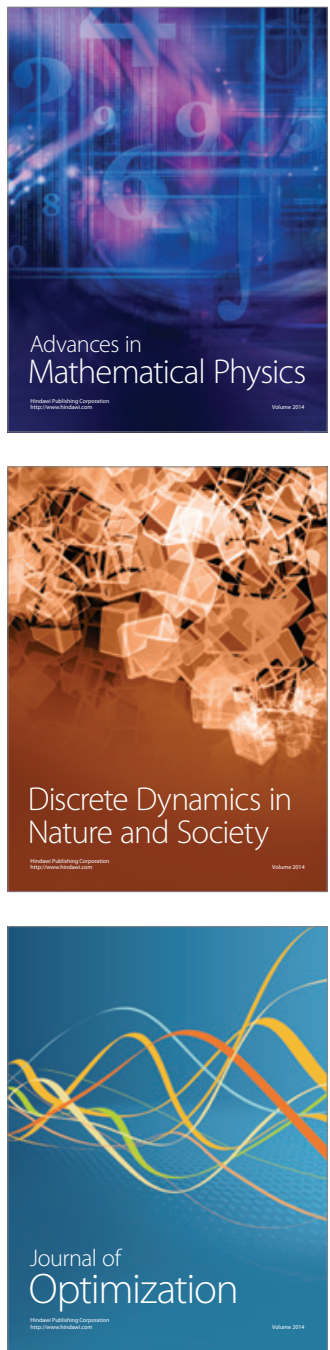